\title{
SYNTHESIS OF SOME NOVEL AND BIOLOGICALLY ACTIVE SCHIFF BASES BEARING A 1,3,4-THIADIAZOLE MOIETY UNDER ACIDIC AND PTC CONDITIONS
}

\author{
A. MOBINIKHALEDI ${ }^{*}{ }^{1}$ M. JABBARPOUR ${ }^{1}$ AND A. HAMTA ${ }^{2}$ \\ ${ }^{1}$ Department of Chemistry, Faculty of Science, Arak University, Arak 38156-8-8349, Iran \\ ${ }^{2}$ Department of Biology, Faculty of Science, Arak University, Arak 38156-8-8349, Iran
}

(Received: March 31, 2011 - Accepted: May 31, 2011)

\begin{abstract}
The synthesis of some new Schiff bases bearing a 1,3,4-thiadiazole moiety, 3a-l, by reaction of 2-amino-5-mercapto-1,3,4-thiadiazole with aromatic aldehydes under acidic and phase transfer catalyst (PTC) conditions was studied. The structure of all the Schiff bases was characterized using FT-IR and NMR spectroscopy, and elemental analyses. The antibacterial activity of these compounds was investigated against Staphylococcus aureus (RTCC, 1885), and Escherichia coli (ATCC, 35922).
\end{abstract}

Key words: Schiff base, 1,3,4-thiadiazole, biological activities, PTC

\section{INTRODUCTION}

There has been considerable attention to the chemistry of Schiff bases because of their wide range of applications in many fields including biological, organic, inorganic and analytical chemistry. They are used as pigments, dyes, catalysts, intermediates in organic and inorganic synthesis and polymer stabilizers. ${ }^{1.4}$ Schiff bases containing heterocyclic rings are known to show cytotoxic, anticonvulsant, antimicrobial, anticancer, antifungal, antimalarial, antiviral, antidepressant and enzyme inhibitor activities..$^{5-11}$ Furthermore, some Schiff bases are used in ion sensors and electrochemical sensors to empower detection with enhanced selectivity and sensitivity. ${ }^{12,13}$

Due to the importance and diverse structural aspects of Schiff bases, several methods and procedures have been reported for synthesis of these compounds. ${ }^{14-19}$ These methods usually involve the straightforward acidcatalyzed reaction of a primary amine and a carbonyl group in organic solvents. However, in spite of their advantages, most of them use procedures in which the chemical safety and yields of products are the main considerations. Hardly ever has attention been concentrated on the fact that some of these established methods use toxic solvents, special apparatus, or strong acid conditions. Consequently, in order to overcome these limitations, the introduction of more efficient methods for the synthesis of Schiff bases is still a challenge. Recently, significant research has been directed toward the solvent-free reaction in order to improve classical procedures and make them cleaner and easier to carry out. ${ }^{20,21}$ Keeping these reports in mind and also due to our interest in the synthesis of Schiff bases ${ }^{16,22-24}$ we wished to study the synthesis of some novel and biologically active Schiff bases bearing a 1,3,4-thiadiazole moiety under both solvent-free and acidic conditions.

\section{EXPERIMENTAL}

All reagents were commercially available without further purification. Melting points were determined with an Electrothermal WRS-1B apparatus. The IR spectra were recorded on a Galaxy FTIR 5000 spectrometer using $\mathrm{KBr}$ discs. The NMR spectra were recorded on a Bruker Avance spectrometer (300 MHz) using DMSO- $d_{6}$ as a solvent and TMS as an internal standard. Microanalyses were performed using the (Elemental, Vario EL III) Elemental Analyzer at Arak University. Reactions were monitored by thin layer chromatography (TLC) using silica gel $\mathrm{F}_{254}$-coated aluminum sheets (Merck). The microbial strains, Staphylococcus aureus (RTCC, 1885) and and Escherichia coli (ATCC, 35922) were obtained from the Pasteur Institute of Iran. The culture medium was Mueller Hinton Agar (MH).

Synthesis of Schiff bases 3 a-l with $\mathrm{H}_{2} \mathrm{SO}_{4}$

To a mixture of 2-amino-5-mercapto-1,3,4-thiadiazole $1(0.133 \mathrm{~g}, 1 \mathrm{mmol})$ and corresponding aromatic aldehyde $\mathbf{2}(1 \mathrm{mmol})$ in ethanol $(10 \mathrm{ml})$ was added 3-4 drops of concentrated sulfuric acid. The reaction mixture was refluxed for an appropriate time as presented in Table 1. After completion of the reaction, controlled by TLC, the reaction mixture was cooled to room temperature giving a solid product after 10-30 min, which was filtered and washed with a mixture of hot water and ethanol $(1: 1,10 \mathrm{ml})$ to afford the pure product 3 .
Synthesis of Schiff bases 3a-l with PTC

A mixture of 2-amino-5-mercapto-1,3,4-thiadiazole 1 (1 mmol), the corresponding aromatic aldehyde $\mathbf{2}(1 \mathrm{mmol})$ and benzyl triethylammonium chloride (BTEAC, $10 \mathrm{~mol} \%$ ) was heated in an oil bath at $90{ }^{\circ} \mathrm{C}$ for an appropriate time as presented in Table 1. The liquid mixture was cooled to room temperature to give a solid mass after 5-20 min, which was washed with $\mathrm{H}_{2} \mathrm{O} / \mathrm{EtOH}(1: 1,10 \mathrm{ml})$ to give the pure product 3 .

\section{Antibacterial study}

The agar diffusion test, or Kirby-Bauer disk-diffusion method was used for this purpose. Each chemically synthesized material $(5 \mathrm{mg})$ was dissolved in $250 \mu \mathrm{l}$ of DMSO and $100 \mu \mathrm{l}$ of the solution of the test compounds was introduced onto the disks $(0.7 \mathrm{~cm}$ diameter $)$. The disks were then placed on top of the medium previously inoculated with the bacteria. $100 \mu \mathrm{l}$ of solvent (DMSO) was added to another disk and implanted as a negative control on each plate along with the standard drugs. The plates were incubated overnight at $37^{\circ} \mathrm{C}$. The inhibition zones were measured and compared with the controls. For the zone size interpretations were used recommendations of the National Committee of Clinical Laboratory Standards (NCCLs). The results are given in Table 2.

2-(Benzylideneamino)-5-mercapto-1,3,4-thiadiazole (3a). m.p 259-261 ${ }^{\circ} \mathrm{C}$. IR (KBr): $v_{\max } 3043\left(\mathrm{CH}_{\text {aromatic }}\right), 1606(\mathrm{C}=\mathrm{N}), 1572(\mathrm{C}=\mathrm{C}) .{ }^{1} \mathrm{H}-\mathrm{NMR}: \delta_{\mathrm{H}}$ $14.56(\mathrm{~s}, 1 \mathrm{H}, \mathrm{S}-\mathrm{H}) 8.73(\mathrm{~s}, 1 \mathrm{H}, \mathrm{CH}=\mathrm{N}), 7.68-7.45(\mathrm{~m}, 5 \mathrm{H}, \mathrm{H}){ }^{13} \mathrm{C}-\mathrm{NMR}$ : $\delta_{\mathrm{C}} 193.5(\mathrm{C}-\mathrm{N}), 169.3\left(\mathrm{C}_{\text {imine }}\right), 135.0(\mathrm{C}-\mathrm{S}), 130.4,129.9,129.5,129.3\left(\mathrm{CH}_{\text {arom }}\right)$. Anal. Calcd for: $\mathrm{C}_{9} \mathrm{H}_{7} \mathrm{~N}_{3} \mathrm{~S}_{2}, \mathrm{C}, 48.85 ; \mathrm{H}, 3.19 ; \mathrm{N}, 18.99 ; \mathrm{S}, 28.98 \%$. Found: $\mathrm{C}$, $48.65 ; \mathrm{H}, 3.01 ; \mathrm{N}, 19.18 ; \mathrm{S}, 29.25 \%$.

2-(2-Hydroxybenzylideneamino)-5-mercapto-1,3,4-thiadiazole (3b). m.p 252-254 ${ }^{\circ} \mathrm{C}$. IR (KBr): $v_{\max } 3070\left(\mathrm{CH}_{\text {rromatic }}\right), 1562(\mathrm{C}=\mathrm{N}) .{ }^{1} \mathrm{H}-\mathrm{NMR}: \delta_{\mathrm{H}}$ $14.54(\mathrm{~s}, 1 \mathrm{H}, \mathrm{S}-\mathrm{H}) 11.14(\mathrm{~s}, 1 \mathrm{H}, \mathrm{OH}), 8.86(\mathrm{~s}, 1 \mathrm{H}, \mathrm{CH}=\mathrm{N}), 7.86-6.95(\mathrm{~m}, 4 \mathrm{H}$, $\left.\mathrm{H}_{\text {aromatic }}\right),{ }^{13} \mathrm{C}-\mathrm{NMR}: \delta_{\mathrm{C}} 192.4(\mathrm{C}-\mathrm{N}), 181.3\left(\mathrm{CH}_{\text {aromatic }}\right), 161.9\left(\mathrm{C}_{\text {imine }}\right), 161.1(\mathrm{C}-$ S), 136.8, 129.9, 122.6, 119.9, $117.6\left(\mathrm{CH}_{\text {aromatic }}\right)$. Anal. Calcd for: $\mathrm{C}_{9} \mathrm{H}_{7} \mathrm{~N}_{3} \mathrm{OS}_{2}$, C, 45.55; H, 2.97; N, 17.71; S, 27.02\%. Found: C, 45.60; H, 2.71; N, 17.95; S, $27.26 \%$.

2-(2-Methoxybenzylideneamino)-5-mercapto-1,3,4-thiadiazole (3c). m.p 148-149 ${ }^{\circ}$ C. IR $(\mathrm{KBr}): v_{\max } 3003\left(\mathrm{CH}_{\text {aromatic }}\right), 2937-2835\left(\mathrm{CH}_{\text {aliphatic }}\right), 1599$ $(\mathrm{C}=\mathrm{N}) .{ }^{1} \mathrm{H}-\mathrm{NMR}: \delta_{\mathrm{H}} 14.51(\mathrm{~s}, 1 \mathrm{H}, \mathrm{S}-\mathrm{H}), 8.82(\mathrm{~s}, 1 \mathrm{H}, \mathrm{CH}=\mathrm{N}), 7.78-7.00(\mathrm{~m}$, $\left.4 \mathrm{H}, \mathrm{H}_{\text {aromatic }}\right), 3.86\left(\mathrm{H}, 3 \mathrm{H}, \mathrm{CH}_{3}\right),{ }^{13} \mathrm{C}-\mathrm{NMR}: \delta_{\mathrm{C}} 189.5(\mathrm{C}-\mathrm{N}), 187.0\left(\mathrm{CH}_{\text {aromatic }}\right)$, $163.4\left(\mathrm{CH}_{\text {imine }}\right), 160.9(\mathrm{C}-\mathrm{S}), 136.4,128.0,122.3,121.4,112.8\left(\mathrm{CH}_{\text {aromatic }}\right), 56.5$ $\left(\mathrm{OCH}_{3}\right)$. Anal. Calcd for: $\mathrm{C}_{10} \mathrm{H}_{0} \mathrm{~N}_{3} \mathrm{OS}, \mathrm{C}, 47.79 ; \mathrm{H}, 3.61 ; \mathrm{N}, 16.72 ; \mathrm{S}, 25.52 \%$. Found: C, 47.96; H, 3.51; N, 16.51; S, 25.26\%.

2-(2-Chlorobenzylideneamino)-5-mercapto-1,3,4-thiadiazole (3d). m.p 242-244 ${ }^{\circ} \mathrm{C}$. IR ( $\left.\mathrm{KBr}\right): v 3068(\mathrm{CH}), 1560(\mathrm{C}=\mathrm{N}) .{ }^{1} \mathrm{H}-\mathrm{NMR}: \delta_{\mathrm{H}} 14.56$ (s, $1 \mathrm{H}, \mathrm{S}-\mathrm{H}), 8.98(\mathrm{~s}, 1 \mathrm{H}, \mathrm{CH}=\mathrm{N}), 7.68-7.46\left(\mathrm{~m}, 4 \mathrm{H}, \mathrm{H}_{\text {aromatic }}\right) .{ }^{13} \mathrm{C}-\mathrm{NMR}: \delta_{\mathrm{C}}$ $190.2(\mathrm{C}-\mathrm{N}), 164.3\left(\mathrm{CH}_{\text {iminim }}\right), 136.1(\mathrm{C}-\mathrm{S}), 131.3,131.1,130.9,130.0,128.4$, $128.3\left(\mathrm{CH}_{\text {aromatic }}\right)$. Anal. Calcd for: $\mathrm{C}_{9} \mathrm{H}_{6} \mathrm{ClN}_{3} \mathrm{~S}_{2}, \mathrm{C}, 42.27 ; \mathrm{H}, 2.36 ; \mathrm{N}, 16.43 ; \mathrm{S}$, $25.08 \%$. Found: C, $42.01 ; \mathrm{H}, 2.30 ; \mathrm{N}, 16.59 ; \mathrm{S}, 25.28 \%$. 
2-(5-Bbromo-2-hydroxybenzylideneamino)-5-mercapto-1,3,4thiadiazole (3e). m.p 254-255 ${ }^{\circ} \mathrm{C}$. IR ( $\left.\mathrm{KBr}\right): v_{\max } 3086(\mathrm{CH}), 1612$ $(\mathrm{C}=\mathrm{N}), 1512(\mathrm{C}=\mathrm{C}) .{ }^{\mathrm{P}} \mathrm{H}-\mathrm{NMR}: \delta_{\mathrm{H}} 14.57(\mathrm{~s}, 1 \mathrm{H}, \mathrm{S}-\mathrm{H}), 11.28(\mathrm{~s}, 1 \mathrm{H}, \mathrm{OH}), 8.79$ $(\mathrm{s}, 1 \mathrm{H}, \mathrm{CH}=\mathrm{N}), 7.98-6.96\left(\mathrm{~m}, 3 \mathrm{H}, \mathrm{H} \quad{ }^{13} \mathrm{C}-\mathrm{NMR}: \delta_{\mathrm{C}} 187.1(\mathrm{C}-\mathrm{N}), 164.4\right.$ $\left(\mathrm{CH}_{\text {aromatic }}\right), 164.2\left(\mathrm{CH}_{\text {imine }}\right), 159.6(\mathrm{C}-\mathrm{S}), 138.2,131.4,122.2,119.8,111.3$ $\left(\mathrm{CH}^{\text {aromatic }}\right)$. Anal. Calcd for: $\mathrm{C}_{0} \mathrm{H}_{6} \mathrm{BrN}_{3} \mathrm{OS}, \mathrm{C}, 34.19 ; \mathrm{H}, 1.91 ; \mathrm{N}, 13.29 ; \mathrm{S}$, $20.28 \%$. Found: C, $34.30 ; \mathrm{H}, 2.21 ; \mathrm{N}, 13.19 ; \mathrm{S}, 20.16 \%$.

2-(3-Bromobenzylideneamino)-5-mercapto-1,3,4-thiadiazole (3f).
235-237 ${ }^{\circ} \mathrm{C}$. IR (KBr): $v 3078(\mathrm{CH}), 1568(\mathrm{C}=\mathrm{N}), 1510(\mathrm{C}=\mathrm{C})$. m.p 235-237 ${ }^{\circ} \mathrm{C}$. IR $(\mathrm{KBr}): v_{\max } 3078\left(\mathrm{CH}_{\text {aromatic }}\right), 1568(\mathrm{C}=\mathrm{N}), 1510(\mathrm{C}=\mathrm{C})$.
${ }^{1} \mathrm{H}-\mathrm{NMR}: \delta 14.62(\mathrm{~s}, 1 \mathrm{H}, \mathrm{S}-\mathrm{H}), 8.72(\mathrm{~s}, 1 \mathrm{H}, \mathrm{CH}=\mathrm{N}), 8.15-7.50(\mathrm{~m}, 4 \mathrm{H}$, $\mathrm{H}{ }) .{ }^{13} \mathrm{C}-\mathrm{NMR}: \delta_{1} 192.3(\mathrm{C}-\mathrm{N}), 137.4\left(\mathrm{CH}_{\text {inin }}\right), 136.8(\mathrm{C}-\mathrm{S}), 132.4,131.7$, 129.9, 129.1, 128.6, $122.8\left(\mathrm{CH}_{\text {aromatic }}\right)$. Anal. Calcd for: $\mathrm{C}_{9} \mathrm{H}_{6} \mathrm{BrN}_{3} \mathrm{~S}_{2}, \mathrm{C}, 36.01$; H, 2.01; N, 14.00; S, 21.36\%. Found: , C, 36.14; H, 2.11; N, 14.09; S, 21.17\%.

2-(4-Isopropylbenzylideneamino)-5-mercapto-1,3,4-thiadiazole (3g). m.p $237-239{ }^{\circ} \mathrm{C}$. IR $(\mathrm{KBr}): v_{\max } 3092\left(\mathrm{CH}_{\text {aromati }}\right), 2960\left(\mathrm{CH}_{\text {aliphati }}\right), 1566$ $(\mathrm{C}=\mathrm{N}) .{ }^{1} \mathrm{H}-\mathrm{NMR}: \delta_{\mathrm{H}} 14.53(\mathrm{~s}, 1 \mathrm{H}, \mathrm{S}-\mathrm{H}), 8.68(\mathrm{~s}, 1 \mathrm{H}, \mathrm{CH}=\mathrm{N}), 7.93-7.43(\mathrm{~m}$, $4 \mathrm{H}, \mathrm{H}$ ) $3.02-2.87(\mathrm{~m}, 1 \mathrm{H}, \mathrm{CH}), 1.23-1.21(\mathrm{dd}, 6 \mathrm{H}, \mathrm{CH}) .{ }^{13} \mathrm{C}-\mathrm{NMR}: \delta_{\mathrm{c}}$ 193.0 (C-N $), 169.0\left(\mathrm{CH}_{\text {imine }}\right), 130.7\left(\mathrm{CH}_{\text {aromatic }}\right), 130.2(\mathrm{C}-\mathrm{S}), 127.6,127.5,127.2$ ( $)$ ), 34.1 (C-Mes), $23.8\left(3 \mathrm{CH}_{3}\right)$. Anal. Calcd for: $\mathrm{C}_{12} \mathrm{H}_{13} \mathrm{~N}_{3} \mathrm{~S}, \mathrm{C}, 54.72$; $\mathrm{H}, 4.97 ; \mathrm{N}, 15.95 ; \mathrm{S}, 24.35 \%$. Found: C, $54.58 ; \mathrm{H}, 4.83 ; \mathrm{N}, 16.21 ; \mathrm{S}, 24.49 \%$.

2-(4-Dimethylaminobenzylideneamino)-5-mercapto-1,3,4-thiadiazole (3h). m.p $246-248{ }^{\circ} \mathrm{C}$. IR (KBr): $v_{\text {max }} 3086\left(\mathrm{CH}_{\text {aromatic }}\right), 2893\left(\mathrm{CH}_{\text {inhatic }}\right), 1591$ $(\mathrm{C}=\mathrm{N}) .{ }^{1} \mathrm{H}-\mathrm{NMR}: \delta_{1} 14.26(\mathrm{~s}, 1 \mathrm{H}, \mathrm{S}-\mathrm{H}), 8.41(\mathrm{~s}, 1 \mathrm{H}, \mathrm{CH}=\mathrm{N}), 7.81-6.79(\mathrm{~m}$, $\left.4 \mathrm{H}, \mathrm{H}_{\text {aromatic }}\right), 3.07\left(\mathrm{~s}, 6 \mathrm{H}, \mathrm{CH}_{3}\right) .{ }^{13} \mathrm{C}-\mathrm{NMR}: \delta_{\mathrm{C}} 186.0(\mathrm{C}-\mathrm{N}), 167.6\left(\mathrm{CH}_{\text {imine }}\right)$, $154.4(\mathrm{CH}), 132.8(\mathrm{C}-\mathrm{S}), 121.5,112.0,111.4(\mathrm{CH}), 65.0\left(\mathrm{CH}^{2}\right)$. Anal. Calcd for: $\mathrm{C}_{11} \mathrm{H}_{12} \mathrm{~N}_{4} \mathrm{~S}_{2}, \mathrm{C}, 49.97 ; \mathrm{H}, 4.58 ; \mathrm{N}, 21.19 ; \mathrm{S}, 24.26 \%$. Found: $\mathrm{C}$, $49.78 ; \mathrm{H}, 4.49 ; \mathrm{N}, 21.36 ; \mathrm{S}, 24.14 \%$.

2-(4-Nitrobenzylideneamino)-5-mercapto-1,3,4-thiadiazole (3i). m.p 259-261 ${ }^{\circ} \mathrm{C}$. IR ( $\left.\mathrm{KBr}\right): v \quad 3014(\mathrm{CH} \quad), 1610(\mathrm{C}=\mathrm{N}), 1568(\mathrm{C}=\mathrm{C}), 1521$, $1352\left(\mathrm{NO}_{2}\right) .{ }^{1} \mathrm{H}-\mathrm{NMR}: \delta_{\mathrm{H}}^{\max } 14.71(\mathrm{~s}, 1 \mathrm{H}, \mathrm{S}-\mathrm{H}), 8.87(\mathrm{~s}, 1 \mathrm{H}, \mathrm{CH}=\mathrm{N}), 8.40-8.22$ $\left(\mathrm{m}, 4 \mathrm{H}, \mathrm{H}_{\text {aromatic }}\right) \cdot{ }^{13} \mathrm{C}-\mathrm{NMR}: \delta_{\mathrm{C}} 181.3(\mathrm{C}-\mathrm{N}), 167.5\left(\mathrm{CH}_{\text {imine }}\right), 131.5\left(\mathrm{CH}_{\text {aromatic }}\right)$, $130.1(\mathrm{C}-\mathrm{S}), 128.8,124.6,124.0(\mathrm{CH})$. Anal. Calcd for: $\mathrm{C}_{9} \mathrm{H}_{6} \mathrm{~N}_{4} \mathrm{O}_{2} \mathrm{~S}_{2}, \mathrm{C}$, 40.59 ; H, 2.27; N, 21.04; S, 24.08\%. Found: 40.71; H, 2.35; N, 21.19; S, $24.17 \%$.

2-(4-Benzyloxybenzylideneamino)-5-mercapto-1,3,4-thiadiazole (3j). mp 215-217 ${ }^{\circ} \mathrm{C}$. IR (KBr): $v_{\text {a }} 3036(\mathrm{CH}), 2982(\mathrm{CH}), 1610(\mathrm{C}=\mathrm{N})$, $1568(\mathrm{C}=\mathrm{C}) .{ }^{1} \mathrm{H}-\mathrm{NMR}: \delta_{\mathrm{H}} 14.46(\mathrm{~s}, 1 \mathrm{H}, \mathrm{S}-\mathrm{H}), 8.62(\mathrm{~s}, 1 \mathrm{H}, \mathrm{CH}=\mathrm{N}), 7.95-7.00$ $\left(\mathrm{m}, 9 \mathrm{H}, \mathrm{H} \quad\right.$ ) $5.22-5.15\left(\mathrm{~d}, 2 \mathrm{H}, \mathrm{CH}_{2}\right) \cdot{ }^{13} \mathrm{C}-\mathrm{NMR}: \delta_{\mathrm{C}} 186.9(\mathrm{C}-\mathrm{N}), 168.3$ $\left(\mathrm{CH}_{\text {aromatic }}\right), 165.3\left(\mathrm{CH}_{\text {imine }}\right), 163.4(\mathrm{C}-\mathrm{S}), 136.7,132.7,128.9,128.5,128.3$, 127.5, 115.9 (CH $), 70.1\left(\mathrm{CH}_{2}\right)$. Anal. Calcd for: $\mathrm{C}_{10} \mathrm{H}_{13} \mathrm{NOS}_{2}, \mathrm{C}, 58.69$; H, 4.00; N, 12.83; S, 19.59\%. Found: C, 58.83; H, 4.11; N, 12.61; S, 19.77\%.

2-(4-Chlorobenzylideneamino)-5-mercapto-1,3,4-thiadiazole (3k). m.p 222-224 ${ }^{\circ} \mathrm{C}$. IR $(\mathrm{KBr}): v_{\max } 3016\left(\mathrm{CH}_{\text {aromatic }}\right), 1610(\mathrm{C}=\mathrm{N}), 1564(\mathrm{C}=\mathrm{C})$. ${ }^{1} \mathrm{H}-\mathrm{NMR}: \delta_{\mathrm{H}} 14.50(\mathrm{~s}, 1 \mathrm{H}, \mathrm{S}-\mathrm{H}), 8.74(\mathrm{~s}, 1 \mathrm{H}, \mathrm{CH}=\mathrm{N}), 7.67-7.47(\mathrm{~m}, 4 \mathrm{H}$, $\mathrm{H}{ }){ }^{13} \mathrm{C}-\mathrm{H} M R: \delta_{\mathrm{C}} 187.0(\mathrm{C}-\mathrm{N}), 168.1\left(\mathrm{CH}_{\mathrm{imin}}\right), 138.8(\mathrm{C}-\mathrm{S}), 133.4,131.9$, 129.8, $129.4\left(\mathrm{CH}_{\mathrm{C}}\right)$. Anal. Calcd for: $\mathrm{C}_{0} \mathrm{H}_{\mathrm{imin}} \mathrm{ClN}_{3} \mathrm{~S}_{2}, \mathrm{C}, 42.27 ; \mathrm{H}, 2.36 ; \mathrm{N}$, $16.43 ;$ S, 25.08\%. Found: $\mathrm{C}, 42.09 ; \mathrm{H}, 2.50 ; \mathrm{N}, 16.11 ; \mathrm{S}, 25.24$

2-(4-Flurobenzylideneamino)-5-mercapto-1,3,4-thiadiazole

(3I). m.p 233-234 ${ }^{\circ} \mathrm{C}$. IR (KBr): $v_{\max } 3009\left(\mathrm{CH}_{\text {aromatic }}\right), 1606(\mathrm{C}=\mathrm{N}), 1564(\mathrm{C}=\mathrm{C})$. ${ }^{1} \mathrm{H}-\mathrm{NMR}: \delta_{\mathrm{H}} 14.54(\mathrm{~s}, 1 \mathrm{H}, \mathrm{S}-\mathrm{H}), 8.73(\mathrm{~s}, 1 \mathrm{H}, \mathrm{CH}=\mathrm{N}), 8.09-7.38(\mathrm{~m}, 4 \mathrm{H}$, $\left.\mathrm{H}_{\text {aromatic }}\right) .{ }^{13} \mathrm{C}-\mathrm{H} M R: \delta_{\mathrm{C}} 187.2(\mathrm{C}-\mathrm{N}), 168.1\left(\mathrm{CH}_{\text {imine }}\right), 167.4\left(\mathrm{C}_{\text {aromatic }}, J_{\mathrm{C}-\mathrm{F}}=240.1\right.$ $\mathrm{Hz}), 133.4(\mathrm{C}-\mathrm{S}), 133.0\left(\mathrm{C}_{\text {rromatic }}, J_{\mathrm{C}-\mathrm{F}}=3.0 \mathrm{~Hz}\right), 129.6\left(\mathrm{C}_{\text {aromatic }}, J_{\mathrm{C}-\mathrm{F}}=7.4 \mathrm{~Hz}\right)$, $116.7(\mathrm{C}, J=20.1 \mathrm{~Hz})$. Anal. Calcd for: $\mathrm{C}_{0} \mathrm{HFN}_{3} \mathrm{~S}_{2}, \mathrm{C}, 45.17 ; \mathrm{H}, 2.53$; $\mathrm{N}, 17.56 ; \mathrm{S}, 26.80 \%$. Found: C, 45.30; H, 2.47; N, 16.21; S, 25.09.

\section{RESULTS AND DISCUSSION}

The synthetic route for the preparation of the target compounds is outlined in Scheme 1.

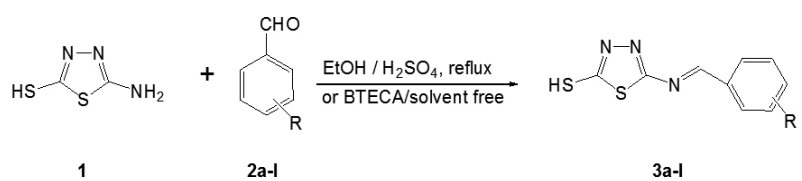

Scheme 1. Preparation of Schiff bases 3a-1
Reaction of 2-amino-5-mercapto-1,3,4-thiadiazole 1 with corresponding aromatic aldehyde $\mathbf{2}$ under acidic conditions or in the presence of BTEAC catalyst afforded 5-mercapto-1,3,4-thiadiazole Schiff bases 3a-l. The reaction was carried out with different aromatic aldehydes bearing electron-withdrawing groups (such as nitro, halide), or electron-releasing groups (such as methyl, $\mathrm{NMe}_{2}$ ). The results are summarized in Table 1 .

Table 1. Synthesis of Schiff bases 3a-I under acidic and solvent free conditions.

\begin{tabular}{|c|c|c|c|c|c|c|}
\hline Entry & $\mathrm{R}$ & Product $^{\mathrm{a}}$ & $\begin{array}{l}\text { Time }^{b} \\
\text { (min) }\end{array}$ & $\begin{array}{c}\text { Yield }^{b} \\
(\%)\end{array}$ & $\begin{array}{l}\text { Time }^{\mathrm{c}} \\
(\mathrm{min})\end{array}$ & $\begin{array}{c}\text { Yield }^{c} \\
(\%)\end{array}$ \\
\hline 1 & $\mathrm{H}$ & $3 a$ & 30 & 65 & 20 & 80 \\
\hline 2 & $2-\mathrm{OH}$ & $3 \mathbf{b}$ & 20 & 71 & 10 & 89 \\
\hline 3 & 2-OMe & $3 c$ & 60 & 40 & 30 & 61 \\
\hline 4 & $2-\mathrm{Cl}$ & 3d & 60 & 56 & ---- & ---- \\
\hline 5 & 2-OH, $5-\mathrm{Br}$ & $3 e$ & 40 & 40 & 30 & 72 \\
\hline 6 & $3-\mathrm{Br}$ & $3 f$ & 20 & 65 & 20 & 75 \\
\hline 7 & 4-Isopropyl & $3 g$ & 30 & 40 & 20 & 71 \\
\hline 8 & 4- $\mathrm{NMe}_{2}$ & $3 \mathrm{~h}$ & 30 & 56 & 10 & 95 \\
\hline 9 & $4-\mathrm{NO}_{2}$ & $3 \mathbf{i}$ & 45 & 30 & 30 & 65 \\
\hline 10 & 4- $\mathrm{OCH}_{2} \mathrm{Ph}$ & $3 \mathbf{j}$ & 60 & 38 & ---- & ---- \\
\hline 11 & $4-\mathrm{Cl}^{2}$ & $3 k$ & 40 & 33 & 35 & 53 \\
\hline 12 & $4-\mathrm{F}$ & 31 & ---- & ----- & 30 & 83 \\
\hline
\end{tabular}

The good yields of products, the use of safe and mild reaction conditions and the shorter reaction time are some advantages of the method for the synthesis of Schiff bases in the presence of BTEAC as compared to that carried out with sulfuric acid.

IR and NMR spectra data as well as elemental analyses are consistent with the expected structures. In the ${ }^{1} \mathrm{H}$ NMR spectra of all the Schiff bases synthesized here, the appearance of singlets at 8.41-8.98 and 14.26-14.71 ppm related to the resonance of vinyl and SH protons, respectively, is good evidence for the expected reactions. Also, In the IR spectra of compounds 3a-I the absence of the absorption related to the $\mathrm{NH}_{2}$ group of the starting material is in support of the reactions having taken place.

The antibacterial screening data revealed that none of the compounds showed antibacterial activity against $E$. coli, as an example of gram negative bacteria (Table 2). Compounds $\mathbf{3 b} \mathbf{b} \mathbf{j}$ and $\mathbf{3 l}$ showed good inhibition against $S$. aureus comparable to penicillin. Also, the anti-S. aureus activity of compounds $\mathbf{3 c}, \mathbf{3 e}$ and $\mathbf{3 h}$ is better than that of the other compounds.

Table 2: Inhibition zone diameter for Schiff bases 3a-l.

\begin{tabular}{|c|c|c|c|}
\hline Entry & Compounds & S. aureus $(\mathrm{mm})$ & E. coli $(\mathrm{mm})$ \\
\hline 1 & 3a & - & - \\
\hline 2 & $\mathbf{3 b}$ & $15 \pm 0.1$ & - \\
\hline 3 & $\mathbf{3 c}$ & $25 \pm 0.2$ & - \\
\hline 4 & $\mathbf{3 d}$ & $7 \pm 0.1$ & - \\
\hline 5 & $\mathbf{3 e}$ & $32 \pm 0.2$ & - \\
\hline 6 & $\mathbf{3 f}$ & $9 \pm 0.1$ & - \\
\hline 7 & $\mathbf{3 g}$ & $8 \pm 0.2$ & - \\
\hline 8 & $\mathbf{3 h}$ & $29 \pm 0.2$ & - \\
\hline 9 & $\mathbf{3 i}$ & $17 \pm 0.1$ & - \\
\hline 10 & $\mathbf{3 j}$ & $17 \pm 0.1$ & - \\
\hline 11 & $\mathbf{3 k}$ & - & - \\
\hline 12 & $\mathbf{3 l}$ & $9 \pm 0.1$ & - \\
\hline 13 & DMSO & - & - \\
\hline Gentamicin & 10 U Penicillin $33 \mathrm{~mm}$ & \multicolumn{2}{|c|}{ Standard drugs } \\
\hline $18 \mathrm{~mm}$ & & \multicolumn{2}{|c|}{} \\
\hline
\end{tabular}

— indicates resistance of bacteria to compounds. The inhibition zone numbers are the averages of three independent experiments. 


\section{ACKNOWLEDGEMENT}

We gratefully acknowledge financial support by the Chemistry Department of Arak University.

\section{References}

1. a) D. N, Dhar, C. L. Taploo, J. Sci. Ind. Res. 41, 501 (1982). b) H-B. Zhu, Z-Y. Dai, W. Haung, K. Cui, Sh-H. Gou, Ch-J. Zhu, Polyhedron. 23, 1131 (2004). c) M. Salavati-Niasari, E. Zamani, M. Bazarganipour, Applied Clay. Sci, 38, 9 (2007). d) M. Colak, N. Demirel, Tetrahedron-Asymmetr. 19,635 (2008).

2. N. Yoshikawa, M. Shibasaki, Tetrahedron. 58, 8289, (2002).

3. a) W. Radecka-Paryzek, V. Patroniak, J. Lisowski, Coord. Chem. Rev. 249, 2156 (2005). b M.Y. Khuhawar, M. A. Mughal, A. H. Channar, Eur. Polym. J. 40, 805 (2004). c) K. C. Gupta, A. K. Sutar, Ch. Lin, Coord. Chem. Rev. 253, 1926 (2009).

4. C. Spînu, M. Pleniceanu, C. Tigae. Turk. J. Chem. 32, 487, (2008).

5. N. Nishat, Sh. Parveen, S. Dhyani, Asma, T. Ahamad.. J. Appl. Polym. Sci. 113, 1671 (2009).

6. Y-Y. Yu, H-D. Xian, J-F. Liu, G-L. Zhao, Molecules, 14, 1747 (2009).

7. A. Jarrahpour, D. Khalili, E. De.Clercq, C. Salmi, J. M. Brunel, Molecules. 12,1720 (2007).

8. a) M. A. Ali, A. H. Mirza, R. J. Butcher, M. T. H. Tarafder, T. B. Keat, A M. Ali, J. Inorg. Biochem. 92, 141 (2002). b) G. B. Bagihalli, P. G. Avaji, S. A. Patil, P. S. Badami, Eur. J. Med. Chem. 43, 2639 (2008).
9. Z. L. You, D. H. Shi, Ch. Xu, Q. Zhang, H. L. Zhu, Eur. J. Med. Chem. 43, 862 (2008).

10 a) N. Rahman, S. Johnson Raja, J. Joseph, A. Sakthivel, J. Dhaveethu Raj, J. Chil. Chem. Soc. 53, 1599, (2008). b) T. Sethi, A. Chaturvedi, R. K. Upadhyay, S. P. Mathur, J. Chil. Chem. Soc. 52, 1206 (2007).

11. Z-L. You, D-H. Shi, H-L.Zhu, Inorg. Chem. Commun. 9, 642 (2006).

12. M. Parra, S. Hernandez, J. Alderete, J. Chil. Chem. Soc. 48 (2003).

13. S. E. Ibrahim, A. Sharif, E-J. Chem. 4, 531 (2007).

14. J. E. dos Santos, E. R. Dockal, E. T. G. Cavalheiro, Carbohydr. Polym. 60, 277 (2005).

15. H. Naeimi, J. Safari, A. Heidarnezhad, Dyes Pigments. 73, 251 (2007)

16 A. Mobinikhaledi, P. J. Steel, M. Polson, Synth. React. Inorg. M. 39, 189 (2009).

17. H. Khanmohammadi, H. Salehifard, M. H. Abnosi, J. Iran. Chem. Soc. 6, 300 (2009).

18. C. Ma, J. Sun, R. Zhang, D. Wang. J. Organomet. Chem. 692, 4029 (2007).

19. R. Zhang, Q. Wang, Q. Li, C. Ma. Inorg. Chim. Acta. 362, 2762 (2009).

20. A. Louri, Top. Curr. Chem. 206, 153 (1999).

21. A. Mobinikhaledi, N. Foroughifar, J. Alipour Safari, E. Amini, J. Heterocyclic Chem. 44, 697 (2007).

22. A. Mobinikhaledi, N. Foroughifar, M. Kalhor, Turk. J. Chem. 34, 367 (2010).

23. A. Mobinikhaledi, N. Foroughifar, M. Kalhor, S. Ebrahimi, Eur. J. Chem. 1, $33(2010)$.

24. A. Mobinikhaledi, M. Kalhor, L. Taheri, Asian J. Chem. 22, 7399 (2010). 